\title{
A Portfolio of Risky Assets and Its Intrinsic Properties
}

\author{
Pierpaolo Angelini \\ Correspondence: Pierpaolo Angelini, Dipartimento di Scienze Statistiche, Universit La Sapienza, Roma, Italy
}

Received: April 7, 2020 Accepted: May 12, 2020 Online Published: May 20, 2020

doi:10.5539/jmr.v12n3p61

URL: https://doi.org/10.5539/jmr.v12n3p61

\begin{abstract}
We show a canonical expression of a univariate risky asset. We find out a canonical expression of the product of two univariate risky assets when they are jointly considered. We find out a canonical expression of a portfolio of two univariate risky assets when it is viewed as a stand-alone entity. We prove that a univariate risky asset is an isometry. We define different distributions of probability on $\mathbb{R}$ inside of metric spaces having different dimensions. We use the geometric property of collinearity in order to obtain this thing. We obtain the expected return on a portfolio of two univariate risky assets when it is viewed as a stand-alone entity. We also obtain its variance. We show that it is possible to use two different quadratic metrics in order to analyze a portfolio of two univariate risky assets. We consider two intrinsic properties of it. If a portfolio of two univariate risky assets is viewed as a stand-alone entity then it is an antisymmetric tensor of order 2 . What we say can be extended to a portfolio of more than two univariate risky assets.
\end{abstract}

Keywords: collinearity, affine tensor, antisymmetric tensor, isometry, $\alpha$-norm, $\alpha$-product

\section{Introduction}

We consider a univariate risky asset denoted by $X=\left\{x^{1}, x^{2}, \ldots, x^{m}\right\}$. We observe that $x^{1}$ is the return on $X$ if $E_{1}$ occurs, $x^{2}$ is the return on $X$ if $E_{2}$ occurs, $\ldots, x^{m}$ is the return on $X$ if $E_{m}$ occurs. We note that $x^{1}$ is the wealth that $X$ yields if $E_{1}$ occurs, $x^{2}$ is the wealth that $X$ yields if $E_{2}$ occurs, $\ldots, x^{m}$ is the wealth that $X$ yields if $E_{m}$ occurs. We say that $E_{1}, E_{2}, \ldots, E_{m}$ are incompatible and exhaustive events of a finite partition of events (Berkelaar, Kouwenberg, \& Post, 2004). We consequently note that $X$ is a univariate random quantity whose possible monetary values denoted by $x^{1}, x^{2}, \ldots, x^{m}$ are all different. One and only one of them will be true a posteriori (de Finetti, 1982a). We assign to each $x^{i}$ of $X$ a subjective probability $p_{i}, i=1, \ldots, m$, where we have $p_{1}+p_{2}+\ldots+p_{m}=1$. We consider a distribution of probability in this way. It is denoted by

$$
\left[\left(x^{1}, p_{1}\right),\left(x^{2}, p_{2}\right), \ldots,\left(x^{m}, p_{m}\right)\right] .
$$

We will also deal with a stand-alone entity coinciding with a portfolio of two univariate risky assets denoted by $X_{12}=$ $\left\{{ }_{1} X,{ }_{2} X\right\}$. We observe that ${ }_{1} X$ and ${ }_{2} X$ are two univariate risky assets. We say that ${ }_{1} X$ and ${ }_{2} X$ are the components of $X_{12}$. We suppose that each of them has $m$ possible monetary values. We say that an inverse linear relationship exists between ${ }_{1} X$ and ${ }_{2} X$. We do not consider only two marginal univariate distributions of probability connected with ${ }_{1} X$ and ${ }_{2} X$ together with their joint distribution. We will also consider another distribution of probability. It derives from the two marginal univariate distributions of probability as well as from the joint distribution. We will summarize it in order to obtain the expected return on $X_{12}$ when it is viewed as a stand-alone entity. We will also obtain its variance. Probability is defined with respect to events. We say that it is not correct to identify events with sets in a systematic way. An event is not always a subset belonging to a larger closed structure containing different subsets of a given nonempty set, so probability is not always a measure. It is not always necessary to transpose the concepts of measure theory into the calculus of probability. There exist situations for which it is correct to say that an event is a single random case coinciding with a well-determined proposition. Such a proposition will be true or false at the right time. Probability viewed as a nonnegative and additive function taking the value 1 on the whole space of events is then a mass. Probability can subjectively be distributed on particular points in the space of a random quantity. It is a linear space. We will realize that these points are real numbers. If a single random case coinciding with a well-determined proposition is contained in a random quantity then it is expressed by a real number. On the other hand, if we say that probability exists on its own because it does not depend on the mental or instinctive evaluations that a given investor makes of a given random event at a given instant with a given set of information then we use a metaphysical notion of probability. If we say that probability exists outside of him in the sense that it does not exist in his own judgment then we use a metaphysical notion of probability. If we say that probability must externally be represented like something acting behind the universe which can be observed according to its own axioms then we use a metaphysical notion of probability. We will realize that it is possible to replace a closed structure represented by a $\sigma$-algebra with another one expressed by a linear subspace over $\mathbb{R}$ because we use a mechanical meaning of probability (Berti, Pratelli, \& Rigo, 2015). Such a meaning is naturally compatible with all axioms of probability (Koopman, 1940). We consequently say that an event has an intrinsic meaning because we do not choose 
a particular orthonormal basis of the linear space under consideration among all its possible orthonormal bases. We have to note a very important point: the criteria for the evaluation of probability can be different (de Finetti, 1982b). However, they lead to an evaluation which is always subjective. This is because an equiprobable judgment is itself subjective. Such a judgment intrinsically characterizes symmetric probability. Concerning frequentist probability, it makes sense that each investor relates probability back to observed frequency only when he specifies the meaning and conditions of this thing. Symmetric probabilities as well as frequentist probabilities are only elements of judgment evaluated by each investor on the basis of his own judgment. Subjective probability results from this necessary judgment. On the other hand, it is not excluded that subjective probability coincides with symmetric probability. It is not even excluded that subjective probability coincides with frequentist probability. We have to note another very important point: we do not say that probability viewed as a mass is not a measure (Good, 1962). Nevertheless, it is not a fixed measure. Different distributions of a unit mass on particular points in a linear space are different measures. We nevertheless believe that such measures must be subjective. In general, events and points are not then bound by an evaluation of probability which is always the same because it is a predetermined evaluation. It follows that probability viewed as a mass can coherently be moved by an investor according to his subjective opinion (Anscombe \& Aumann, 1963).

\section{A Mathematical Definition of a Univariate Risky Asset}

We consider an $m$-dimensional linear space over $\mathbb{R}$ provided with a Euclidean metric on it. We denote it by $E^{m}$. Given an orthonormal basis of $E^{m}$ denoted by $\left\{\mathbf{e}_{j}\right\}, j=1, \ldots, m$, any $m$-dimensional vector of $E^{m}$ is uniquely expressed by means of a linear combination of basis vectors. We write

$$
\mathbf{x}=x^{1} \mathbf{e}_{1}+x^{2} \mathbf{e}_{2}+\ldots+x^{m} \mathbf{e}_{m}
$$

as well as

where we have

$$
\mathbf{x}=x^{i} \mathbf{e}_{i},
$$

$$
\mathbf{x}=\left(\begin{array}{c}
x^{1} \\
x^{2} \\
\vdots \\
x^{m}
\end{array}\right) \in E^{m}
$$

We suppose that it turns out to be $x^{i} \neq x^{j}, i, j=1, \ldots, m$, so we are able to write $x^{1}<x^{2}<\ldots<x^{m}$ without loss of generality. Also, it is possible to show that each contravariant component of $\mathbf{x} \in E^{m}$ can be viewed as an $m$-dimensional vector of $E^{m}$ denoted by ${ }_{(i)} \mathbf{x}, i=1, \ldots, m$. We therefore write

$$
{ }_{(1)} \mathbf{x}=x^{1} \mathbf{e}_{1},
$$

with $x^{1} \in \mathbb{R}$, as well as

$$
{ }_{(m)} \mathbf{x}=x^{m} \mathbf{e}_{m},
$$

with $x^{m} \in \mathbb{R}$. We observe that ${ }_{(1)} \mathbf{x}$ and $\mathbf{e}_{1}$ are collinear as well as ${ }_{(m)} \mathbf{x}$ and $\mathbf{e}_{m}$. It follows that it turns out to be

$$
{ }_{(1)} \mathbf{x}+\ldots+{ }_{(m)} \mathbf{x}=\mathbf{x}
$$

where each ${ }_{(i)} \mathbf{x}$ is an element of an one-dimensional subspace of $E^{m}$ denoted by $E_{(i)}^{m}, i=1, \ldots, m$. We write

$$
E_{(1)}^{m} \oplus \ldots \oplus E_{(m)}^{m}=E^{m}
$$

because the direct sum of $m$ one-dimensional subspaces of $E^{m}$ coincides with $E^{m}$ itself. We note that this direct sum is also orthogonal. We write

$$
\operatorname{dim} E_{(1)}^{m}+\ldots+\operatorname{dim} E_{(m)}^{m}=\operatorname{dim} E^{m}
$$

where we have $\operatorname{dim} E^{m}=m$. The contravariant components of ${ }_{(i)} \mathbf{x}$ are given by

$$
{ }_{(i)} \mathbf{x}={ }_{(i)} x^{i} \delta_{i}^{j},
$$

where we have $i=1, \ldots, m$. We note that $\delta_{i}^{j}$ denotes the Kronecker delta. If it turns out to be $i=j$ then we have $\delta_{i}^{j}=1$. If it turns out to be $i \neq j$ then we have $\delta_{i}^{j}=0$. We note that (10) is characterized by the Einstein summation convention. Thus, we are able to write

$$
{ }_{(i)} \mathbf{x}=\left(\begin{array}{c}
{ }_{(i)} x^{1} \delta_{1}^{1}+{ }_{(i)} x^{2} \delta_{2}^{1}+\ldots+{ }_{(i)} x^{m} \delta_{m}^{1} \\
{ }_{(i)} x^{1} \delta_{1}^{2}+{ }_{(i)} x^{2} \delta_{2}^{2}+\ldots+{ }_{(i)} x^{m} \delta_{m}^{2} \\
\vdots \\
{ }_{(i)} x^{1} \delta_{1}^{m}+{ }_{(i)} x^{2} \delta_{2}^{m}+\ldots+{ }_{(i)} x^{m} \delta_{m}^{m}
\end{array}\right),
$$


where we have $i=1, \ldots, m$. Having said that, we are able to consider $m$ oriented straight lines of $E^{m}$ which are measured in the same unit of length (von Neumann, 1936). They are pairwise orthogonal. The point where they meet is the origin of $E^{m}$. It is the zero vector of $E^{m}$. We do not consider particular $m$-tuples of real numbers belonging to every straight line of $E^{m}$ but we consider only real numbers connected with each of them. This thing results from the geometric property of collinearity that we have considered. We consequently say that a univariate risky asset denoted by $X$ is a finite partition of incompatible and exhaustive events generically denoted by $E_{1}, E_{2}, \ldots, E_{m}$ such that one and only one of them will be true a posteriori (Tversky, \& Kahneman, 1992). We evidently realize that $X$ is a univariate random quantity (He, \& Zhou, 2011). Each possible monetary value of $X=\left\{x^{1}, x^{2}, \ldots, x^{m}\right\}$ is associated with a single random event. Each possible monetary value of $X$ belongs to one of $m$ straight lines of $E^{m}$. Each straight line of $E^{m}$ represents the whole of the space of alternatives (whose number is infinite) with respect to one of $m$ alternatives of $X$. Each point on a straight line of $E^{m}$ corresponds to a single and possible alternative of $X$ and vice versa. Concerning one of $m$ alternatives of $X$ we observe that information and knowledge of a given investor at a given instant permit him of not to excluding a real number only (deGroot, 1962). It remains possible for him because it is not either true or false. We evidently consider a limitation of expectations in this way (Piccinato, 1986). The same thing goes by thinking of all other alternatives of $X$.

\section{A Canonical Expression of A Univariate Risky Asset}

We have realized that all events contained in $X$ are embedded in $E^{m}$ (de Finetti, 1972a). An event always coincides with a well-determined proposition. An event contained in $X$ coincides with a well-determined proposition expressed by a real number (de Finetti, 1980). Probability meant as a mass is defined inside of a metric space. The probability of an event is contained in the prevision or expected value or mathematical expectation of a random quantity. The notion of prevision of a random quantity is a unique notion (Berti, Regazzini, \& Rigo, 2001). It is called probability in the case of events. Hence, the same symbol $\mathbf{P}$ is used in order to denote both the prevision of a random quantity and the probability of an event (Coletti, Scozzafava, \& Vantaggi, 2015). Anyway, we deal with $m$ masses denoted by $p_{1}, p_{2}, \ldots, p_{m}$ such that we write $p_{1}+p_{2}+\ldots+p_{m}=1$ (de Finetti, 1972b). They are located on $m$ components denoted by $x^{1}, x^{2}, \ldots, x^{m}$ of $m$ vectors denoted by ${ }_{(1)} \mathbf{x},{ }_{(2)} \mathbf{x}, \ldots,{ }_{(m)} \mathbf{x}$ of $E^{m}$. We consider a distribution of probability on $\mathbb{R}$ inside of $E^{m}$ in this way (de Finetti, 1964). This is because $x^{1}, x^{2}, \ldots, x^{m}$ are real numbers. We have evidently $\left\{x^{1}\right\} \in \mathbb{R}$, with ${ }_{(1)} \mathbf{x}=x^{1} \mathbf{e}_{1} \in E_{(1)}^{m}, \ldots,\left\{x^{m}\right\} \in \mathbb{R}$, with ${ }_{(m)} \mathbf{x}=x^{m} \mathbf{e}_{m} \in E_{(m)}^{m}$. After writing

$$
\mathbf{w}=x^{1}\left|E_{1}\right| \mathbf{e}_{1}+x^{2}\left|E_{2}\right| \mathbf{e}_{2}+\ldots+x^{m}\left|E_{m}\right| \mathbf{e}_{m},
$$

with $\mathbf{w} \in E^{m}$, where $\left\{\mathbf{e}_{j}\right\}, j=1, \ldots, m$, is an orthonormal basis of $E^{m}$, it turns out to be

$$
X=x^{1}\left|E_{1}\right|+x^{2}\left|E_{2}\right|+\ldots+x^{m}\left|E_{m}\right|,
$$

where we have

$$
\left|E_{i}\right|= \begin{cases}1, & \text { if } E_{i} \text { is true } \\ 0, & \text { if } E_{i} \text { is false }\end{cases}
$$

for every $i=1, \ldots, m$. We consider $m$ elementary events (constituents) of a finite partition of incompatible and exhaustive events. They are generically denoted by $E_{1}, E_{2}, \ldots, E_{m}$. We consequently observe that $X$ is an identity function such that it is possible to write

$$
i d_{\mathbb{R}}: \mathbb{R} \rightarrow \mathbb{R},
$$

where $\mathbb{R}$ is a linear space over itself and it is of dimension 1 . We say that $X$ is a linear operator whose canonical expression coincides with (13). We say that $X$ is an isometry. It follows that each single event could uniquely be expressed by infinite numbers, so we could also write $\left\{x^{1}+a, x^{2}+a, \ldots, x^{m}+a\right\}$, where $a \in \mathbb{R}$ is an arbitrary constant. This means that we consider infinite translations in this way. We consider different quantities from a geometric viewpoint. They are nevertheless the same quantity from a randomness viewpoint because events and probabilities associated with them do not change. On the other hand, if two or more than two propositions can express the same event contained in $X$ then two or more than two real numbers can uniquely identify it. We say that a change of origin is inessential from a randomness viewpoint. On the other hand, we are always able to lead back the changed origin to the starting one. Hence, we consider a different closed structure in this way. Such a structure is not a $\sigma$-algebra but it is a linear subspace over $\mathbb{R}$. We deal with $m$ subspaces of dimension 1 because every event contained in $X$ belongs to one of them according to (12). On the other hand, a univariate risky asset $X=\left\{x^{1}, x^{2}, \ldots, x^{m}\right\}$ viewed as an $m$-dimensional vector of $E^{m}$ is an element of a set of univariate risky assets denoted by ${ }_{(1)} S$. We note that it turns out to be

$$
{ }_{(1)} S \subset E^{m},
$$

where ${ }_{(1)} S$ is an $m$-dimensional linear space contained in $E^{m}$. This is because the sum of two vectors belonging to ${ }_{(1)} S$ must be a vector whose components are all different. Thus, it belongs to ${ }_{(1)} S$ in this way. We say that it belongs to ${ }_{(1)} S$ 
if and only if its components are all different. The same thing goes when we consider the multiplication of a vector of ${ }_{(1)} S$ by a real number that is different from zero. Hence, we say that ${ }_{(1)} S$ is closed with respect to the sum of two vectors of it and the multiplication of a vector of it by a real number that is different from zero. We consider a closed structure coinciding with an $m$-dimensional linear space contained in $E^{m}$ in this way. We note that $E^{m}$ can also be viewed as an affine space over itself. Each element of $E^{m}$ is firstly an $m$-dimensional vector viewed as an ordered list of $m$ real numbers. Nevertheless, each element of $E^{m}$ can also be viewed as a point of an affine space, where the zero vector of $E^{m}$ is the origin of it. Thus, the zero vector of $E^{m}$ characterizes an affine frame of $E^{m}$ when it is viewed as an affine space. An affine frame of $E^{m}$ viewed as an affine space consists of a point coinciding with the zero vector of $E^{m}$ and an orthonormal basis of $E^{m}$. We are able to consider a point of an affine space having $m$ coordinates or a vector of a linear space having $m$ components. We choose a covariant notation with respect to the components of $\mathbf{p} \in E^{m}$, so we write

$$
\mathbf{p}=\left(\begin{array}{c}
p_{1} \\
p_{2} \\
\vdots \\
p_{m}
\end{array}\right),
$$

where $p_{i}$ represents a subjective probability assigned to $x^{i}, i=1, \ldots, m$, by a given investor according to his degree of belief in the occurrence of $x^{i}$ (Pfanzagl, 1967). We note that it turns out to be $\sum_{i=1}^{m} p_{i}=1$ because we consider a coherent evaluation of the probabilities associated with every single event (Zank, 2010). It is finitely additive (Nunke, \& Savage, 1952) Different investors whose state of knowledge is hypothetically identical may choose different $p_{i}$ whose sum is equal to 1 (Coletti, Petturiti, \& Vantaggi, 2014). Indeed, each of them may subjectively give greater attention to certain circumstances than to others. In any case, if we write

$$
(\mathbf{x}, \mathbf{p}) \subset E^{m}
$$

then we identify a distribution of probability embedded in a linear space provided with a Euclidean metric on it. Such a distribution can always vary from investor to investor (de Finetti, 1989). Moreover, it can also vary with respect to the state of information of a given investor. A coherent prevision of $X$ is given by

$$
\mathbf{P}(X)=x^{1} \mathbf{P}\left(E_{1}\right)+x^{2} \mathbf{P}\left(E_{2}\right)+\ldots+x^{m} \mathbf{P}\left(E_{m}\right) .
$$

It is linear and homogeneous. It is the expected return on the univariate risky asset under consideration (Slovic, Fischhoff, $\&$ Lichtenstein, 1977). From $\mathbf{P}\left(E_{i}\right)=p_{i}, i=1, \ldots, m$, it follows that it turns out to be

$$
\mathbf{P}(X)=x^{1} p_{1}+x^{2} p_{2}+\ldots+x^{m} p_{m} .
$$

We have to note a very important point: we should exactly speak about components of $\mathbf{x}$ and $\mathbf{p}$ having upper and lower indices because we deal with an orthonormal basis of $E^{m}$. It is consequently possible to show that the covariant components of every $m$-dimensional vector of $E^{m}$ coincide with the contravariant ones. We use these terms because we are able to stress that $\mathbf{x}$ and $\mathbf{p}$ are of a different nature in this way. This is because the possible values of $X$ are objective unlike any evaluation of the probabilities associated with every single event connected with $\left\{x^{1}, x^{2}, \ldots, x^{m}\right\}$.

\section{A Canonical Expression of the Product of Two Univariate Risky Assets}

Given a portfolio of two univariate risky assets denoted by $X_{12}=\left\{{ }_{1} X,{ }_{2} X\right\}$, we observe that ${ }_{1} X$ and ${ }_{2} X$ are two marginal univariate risky assets whose possible monetary values are

$$
{ }_{1} X=\left\{{ }_{(1)} x^{1},{ }_{(1)} x^{2}, \ldots,{ }_{(1)} x^{m}\right\}
$$

and

$$
{ }_{2} X=\left\{{ }_{(2)} x^{1},{ }_{(2)} x^{2}, \ldots,{ }_{(2)} x^{m}\right\} .
$$

They are the components of $X_{12}$. We write

$$
{ }_{(1)} \mathbf{x}=\left(\begin{array}{c}
(1) \\
x^{1} \\
(1) \\
x^{2} \\
\vdots \\
(1) x^{m}
\end{array}\right) \in E^{m}
$$

as well as

$$
{ }_{(2)} \mathbf{x}=\left(\begin{array}{c}
(2)^{x^{1}} \\
{ }_{(2)} x^{2} \\
\vdots \\
(2) x^{m}
\end{array}\right) \in E^{m}
$$


where $\left\{\mathbf{e}_{j}\right\}, j=1, \ldots, m$, is again an orthonormal basis of $E^{m}$. We note that each ${ }_{(1)} x^{i}$ is associated with ${ }_{(1)} E_{i}, i=1, \ldots, m$, while each ${ }_{(2)} x^{i}$ is associated with ${ }_{(2)} E_{i}, i=1, \ldots, m$, where

$$
\left\{{ }_{(1)} E_{1},{ }_{(1)} E_{2}, \ldots,{ }_{(1)} E_{m}\right\}
$$

as well as

$$
\left\{{ }_{(2)} E_{1},{ }_{(2)} E_{2}, \ldots,{ }_{(2)} E_{m}\right\}
$$

are two finite partitions of incompatible and exhaustive events. They are elementary events. Having said that, we jointly consider ${ }_{1} X$ and ${ }_{2} X$, so we write

$$
T=\left.{ }_{(1)} x^{1}{ }_{(2)} x^{1}\right|_{(1)} E_{1}\left\|_{(2)} E_{1}\left|\mathbf{e}_{1} \otimes \mathbf{e}_{1}+{ }_{(1)} x^{1}{ }_{(2)} x^{2}\right|_{(1)} E_{1}\right\|_{(2)} E_{2}\left|\mathbf{e}_{1} \otimes \mathbf{e}_{2}+\ldots+{ }_{(1)} x^{m}{ }_{(2)} x^{m}\right|_{(1)} E_{m} \|_{(2)} E_{m} \mid \mathbf{e}_{m} \otimes \mathbf{e}_{m},
$$

where $T \in E^{m} \otimes E^{m}=E_{(2)}^{m}$ is an affine tensor of order 2. We have to note a very important point: we are indifferently able to consider either $\left({ }_{1} X,{ }_{2} X\right)$ or $\left({ }_{2} X,{ }_{1} X\right)$. We consider $\left({ }_{1} X,{ }_{2} X\right)$, so

$$
\left\{{ }_{(1)} E_{1} \wedge{ }_{(2)} E_{1},{ }_{(1)} E_{1} \wedge{ }_{(2)} E_{2}, \ldots,{ }_{(1)} E_{m} \wedge{ }_{(2)} E_{m}\right\}
$$

is a finite partition of incompatible and exhaustive events. They are elementary events. They are overall $\mathrm{m}^{2}$. We observe that $\left\{\mathbf{e}_{i} \otimes \mathbf{e}_{j}\right\}, i, j=1, \ldots, m$, is an orthonormal basis of $E^{m} \otimes E^{m}$. There are $m^{2}$ summands in (27) because we have $\operatorname{dim}\left(E^{m} \otimes E^{m}\right)=m^{2}$. On the other hand, each $\mathbf{e}_{i} \otimes \mathbf{e}_{j}, i, j=1, \ldots, m$, is always an $m \times m$ matrix which is isomorphic to an $m^{2}$-dimensional basis vector of $E^{m^{2}}$. We consider $m^{2}$ straight lines of $E^{m^{2}}$ in this way. They are pairwise orthogonal. They are the axes of the coordinate system under consideration. We are able to consider a distribution of probability on $\mathbb{R}$ inside of $E^{m^{2}}$ in this way. We use the geometric property of collinearity with respect to $E^{m^{2}}$. This means that we deal with $m^{2}$ masses located on $m^{2}$ components denoted by ${ }_{(1)} x_{(2)}^{i} x^{j}, i, j=1, \ldots, m$, of $m^{2}$ vectors of $E^{m^{2}}$. We note that each ${ }_{(1)} x_{(2)}^{i} x^{j}, i, j=1, \ldots, m$, is a real number. A canonical expression of the product of two risky assets ${ }_{1} X$ and ${ }_{2} X$ is given by

$$
{ }_{1} X X_{2} X=\left.{ }_{(1)} x^{1}{ }_{(2)} x^{1}\right|_{(1)} E_{1}\left\|_{(2)} E_{1}\left|+{ }_{(1)} x^{1}{ }_{(2)} x^{2}\right|_{(1)} E_{1}\right\|_{(2)} E_{2}\left|+\ldots+{ }_{(1)} x^{m}{ }_{(2)} x^{m}\right|_{(1)} E_{m} \|_{(2)} E_{m} \mid,
$$

where we have

$$
\left|E_{i}\right|\left|E_{j}\right|= \begin{cases}1, & \text { if } E_{i} \text { and } E_{j} \text { are both true } \\ 0, & \text { otherwise }\end{cases}
$$

for every $i, j=1, \ldots, m$. A coherent prevision of the product of two risky assets ${ }_{1} X$ and ${ }_{2} X$ is then expressed by

$$
\mathbf{P}\left({ }_{1} X_{2} X\right)={ }_{(1)} x_{(2)}^{1} x^{1} \mathbf{P}\left(_{(1)} E_{1} \wedge{ }_{(2)} E_{1}\right)+{ }_{(1)} x_{(2)}^{1} x^{2} \mathbf{P}\left(_{(1)} E_{1} \wedge{ }_{(2)} E_{2}\right)+\ldots+{ }_{(1)} x^{m}{ }_{(2)} x^{m} \mathbf{P}\left(_{(1)} E_{m} \wedge{ }_{(2)} E_{m}\right) .
$$

It is bilinear and homogeneous with respect to $E^{m} \otimes E^{m}$. We say that it is an $\alpha$-product between ${ }_{(1)} \mathbf{x}$ and ${ }_{(2)} \mathbf{x}$ denoted by ${ }_{(1)} \mathbf{x} \odot_{(2)} \mathbf{x}$. From $\mathbf{P}\left(_{(1)} E_{i} \wedge_{(2)} E_{j}\right)=p_{i j}, i, j=1, \ldots, m$, where we have $\sum_{i=1}^{m} \sum_{j=1}^{m} p_{i j}=1$, it follows that it turns out to be

$$
\mathbf{P}\left({ }_{1} X_{2} X\right)={ }_{(1)} x^{1}{ }_{(2)} x^{1} p_{11}+{ }_{(1)} x^{1}{ }_{(2)} x^{2} p_{12}+\ldots+{ }_{(1)} x^{m}{ }_{(2)} x^{m} p_{m m},
$$

so it is possible to write

$$
\operatorname{Cov}\left({ }_{1} X,{ }_{2} X\right)=\mathbf{P}\left({ }_{1} X{ }_{2} X\right)-\mathbf{P}\left({ }_{1} X\right) \mathbf{P}\left({ }_{2} X\right)
$$

where $\mathbf{P}\left({ }_{1} X\right)$ is a coherent prevision of ${ }_{1} X$, while $\mathbf{P}\left({ }_{2} X\right)$ is a coherent prevision of ${ }_{2} X$. A coherent prevision of ${ }_{1} X_{2} X$ denoted by $\mathbf{P}\left({ }_{1} X_{2} X\right)$ can also be expressed by

$$
{ }_{1} \bar{x}_{2} \bar{x}={ }_{(1)} \bar{x}_{(2)}^{i_{1}} \bar{x}^{i_{2}} \mathbf{e}_{i_{1}} \otimes \mathbf{e}_{i_{2}},
$$

where $\bar{x}_{2} \bar{x}$ is an affine tensor of order 2 whose contravariant components are all equal. They coincide with $\mathbf{P}\left({ }_{1} X_{2} X\right)=$ ${ }_{1} \bar{X}_{2} \bar{X}$, where $\mathbf{P}\left({ }_{1} X_{2} X\right)={ }_{1} \bar{X}_{2} \bar{X}$ has evidently been obtained by means of $p=\left(p_{i_{1} i_{2}}\right)=\left(p_{i j}\right)$. This is because we speak about an $\alpha$-product between ${ }_{(1)} \mathbf{x}$ and ${ }_{(2)} \mathbf{x}$. We have to note another very important point: when we write $\mathbf{P}\left({ }_{(1)} E_{i} \wedge{ }_{(2)} E_{j}\right)=$ $p_{i j}, i, j=1, \ldots, m$, we mean that only a subjective and coherent evaluation of probability is always required (Gilio, \& Sanfilippo, 2014). No concept concerning probability can therefore be imposed on probability (Pompilj, 1957). We note that it is possible to measure the degree of belief of a given investor in the occurrence of a random event by using the notion of fair bet on it (Coletti, Petturiti, \& Vantaggi, 2016a). It is then possible to observe which real or conceptual bets on it are judged to be fair by him at a given instant (Coletti, Petturiti, \& Vantaggi, 2016b). We note that those bets in which a given investor with a given set of information at a given instant accepts to be either bettor or bookmaker are judged to be fair by him. In particular, we always consider an investor who hypothetically bets one dollar on different events denoted by ${ }_{(1)} E_{i} \wedge{ }_{(2)} E_{j}, i, j=1, \ldots, m$, belonging to a finite partition of incompatible and exhaustive events. Hence, all these bets are fair when and only when the certain gain denoted by $\sum_{i=1}^{m} \sum_{j=1}^{m} p_{i j}$ that he judges to be equivalent to a unit gain conditional on the occurrence of $\sum_{i=1}^{m} \sum_{j=1(1)}^{m} E_{i} \wedge{ }_{(2)} E_{j}$ is equal to 1 (de Finetti, 1981). 


\section{A Portfolio of Two Univariate Risky Assets Identified With an Antisymmetric Tensor and Its Expected Return}

We denote by $X_{12}=\left\{{ }_{1} X,{ }_{2} X\right\}$ a portfolio of two univariate risky assets belonging to ${ }_{(2)} S^{(2)}$, where ${ }_{(2)} S^{(2)}$ is a set of portfolios of two univariate risky assets. We note that it turns out to be

$$
{ }_{(2)} S^{(2)} \subset E^{m} \otimes E^{m},
$$

so we realize that ${ }_{(2)} S^{(2)}$ is a linear space contained in $E^{m} \otimes E^{m}$. If we write

$$
T=\left.{ }_{(1)} x^{i_{1}}{ }_{(2)} x^{i_{2}}\right|_{(1)} E_{i_{1}} \|_{(2)} E_{i_{2}} \mid \mathbf{e}_{i_{1}} \otimes \mathbf{e}_{i_{2}}
$$

then we deal with an ordered pair of two univariate risky assets denoted by $\left({ }_{1} X,{ }_{2} X\right)$. We have to observe that ${ }_{1} X$ and ${ }_{2} X$ are firstly two quantities, although they are two random quantities. If we conversely consider $\left({ }_{2} X,{ }_{1} X\right)$ then we have to write

$$
T=\left.{ }_{(2)} x^{i_{2}}{ }_{(1)} x^{i_{1}}\right|_{(2)} E_{i_{2}} \|_{(1)} E_{i_{1}} \mid \mathbf{e}_{i_{2}} \otimes \mathbf{e}_{i_{1}} .
$$

We obtain the same tensor denoted by $T$ characterized by different contravariant components in this way. The contravariant components of $T$ expressed by (37) are not the same of the ones expressed by (36). They depend on the ordered pair of univariate risky assets that could be considered. We have consequently to consider (36) and (37) together in order to release a tensor representation of $X_{12}$ from any ordered pair of univariate risky assets which could be considered, $\left.{ }_{1} X,{ }_{2} X\right)$ or $\left({ }_{2} X,{ }_{1} X\right)$. If we express $T$ by considering both (36) and (37) then we observe that $m$ of $m^{2}$ contravariant components are always equal. It is therefore possible to say that the possible values of $X_{12}$ can be expressed by means of the contravariant components of an antisymmetric tensor of order 2 . It is then given by

$$
T=\sum_{i_{1}<i_{2}}\left[\left(\left._{(1)} x^{i_{1}}{ }_{(2)} x^{i_{2}}\right|_{(1)} E_{i_{1}}\left\|_{(2)} E_{i_{2}}\left|-{ }_{(1)} x^{i_{2}}{ }_{(2)} x^{i_{1}}\right|_{(1)} E_{i_{2}}\right\|_{(2)} E_{i_{1}} \mid\right)\right] \mathbf{e}_{i_{1}} \otimes \mathbf{e}_{i_{2}} .
$$

We say that (38) is a canonical expression of $X_{12}$ when it is viewed as a stand-alone entity. Having said that, we denote by ${ }_{12} f$ an antisymmetric tensor of order 2 identifying the possible values of $X_{12}$. The contravariant components of ${ }_{12} f$ are then expressed by

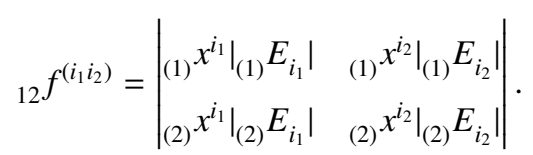

These components are equal to 0 when they have equal contravariant indices. They are then equal to 0 when it turns out to be $i_{1}=i_{2}$. They are not contained in (39) for this reason. We have always $i_{1}<i_{2}$ in (39), with $i_{1}, i_{2} \in I_{m}=\{1, \ldots, m\}$. It follows that the antisymmetric tensors of order 2 identify a linear space whose dimension is equal to $\left(\begin{array}{c}m \\ 2\end{array}\right)$, where we have

$$
\left(\begin{array}{c}
m \\
2
\end{array}\right)<m^{2}
$$

We have to speak about probabilities in order to define $X_{12}$ without shortcomings. We firstly confirm that the tensor of the joint probabilities denoted by $p=\left(p_{i_{1} i_{2}}\right)$ is an affine tensor of order 2. Its covariant components are overall $\mathrm{m}^{2}$. They represent those subjective probabilities connected with the ordered pairs of contravariant components of vectors identifying the marginal univariate risky assets ${ }_{1} X$ and ${ }_{2} X$ of $X_{12}$. We secondly consider those vector homographies that allow us to pass from the contravariant components of a type of vector to the covariant components of another type of vector by means of $p=\left(p_{i_{1} i_{2}}\right)$. We are therefore able to define the covariant components of ${ }_{12} f$ in this way. In analytic terms one has

$$
{ }_{(1)} x^{i_{1}} p_{i_{1} i_{2}}={ }_{(1)} x_{i_{2}}
$$

and

$$
\text { (2) } x^{i_{2}} p_{i_{1} i_{2}}={ }_{(2)} x_{i_{1}}
$$

by virtue of a specific convention that we introduce: if the covariant indices to right-hand side of (41) and (42) vary over all their possible values then we obtain two sequences of values whose elements are overall $(m+m)$. They identify those products between all contravariant components of an $m$-dimensional vector representing a univariate risky asset of $X_{12}$ and a fixed contravariant component of the other $m$-dimensional vector, where all these components are considered together with their probabilities. It turns out to be

$$
{ }_{12} f_{\left(i_{1} i_{2}\right)}=\left|\begin{array}{ll}
{ }_{(1)} x_{i_{1}} & { }_{(1)} x_{i_{2}} \\
{ }_{(2)} x_{i_{1}} & { }_{(2)} x_{i_{2}}
\end{array}\right|=\left|\begin{array}{ll}
{ }_{(1)^{i_{2}}} p_{i_{2} i_{1}} & { }_{(1)} x^{x_{1}} p_{i_{1} i_{2}} \\
(2)^{x_{2}} p_{i_{2} i_{1}} & { }_{(2)} x^{i_{1}} p_{i_{1} i_{2}}
\end{array}\right| .
$$


For instance, we obtain

$$
{ }_{(1)} x^{i_{1}} p_{i_{1} i_{2}}={ }_{(1)} x^{1}{ }_{(2)} x^{1} \mathbf{P}\left({ }_{(1)} E_{1} \wedge{ }_{(2)} E_{1}\right)+{ }_{(1)} x^{2}{ }_{(2)} x^{1} \mathbf{P}\left({ }_{(1)} E_{2} \wedge{ }_{(2)} E_{1}\right)+\ldots+{ }_{(1)} x^{m}{ }_{(2)} x^{1} \mathbf{P}\left({ }_{(1)} E_{m} \wedge{ }_{(2)} E_{1}\right),
$$

where all these events are incompatible. We consequently continue to deal with $m^{2}$ masses located on $m^{2}$ components denoted by $_{(1)} x_{(2)}^{i_{1}} x^{i_{2}}, i_{1}, i_{2} \in I_{m}=\{1, \ldots, m\}$, of $m^{2}$ vectors of $E^{m^{2}}$ in this way. The covariant indices of the tensor $p$ can be interchanged, so it is also possible to write

$$
{ }_{(1)} x^{i_{1}} p_{i_{1} i_{2}}={ }_{(1)} x^{i_{1}} p_{i_{2} i_{1}} .
$$

We are able to compute the $\alpha$-norm of the tensor ${ }_{12} f$ after defining $X_{12}$. It is given by

$$
\left\|_{12} f\right\|_{\alpha}^{2}={ }_{12} f \odot{ }_{12} f={ }_{12} f^{\left(i_{1} i_{2}\right)}{ }_{12} f_{\left(i_{1} i_{2}\right)},
$$

so it turns out to be

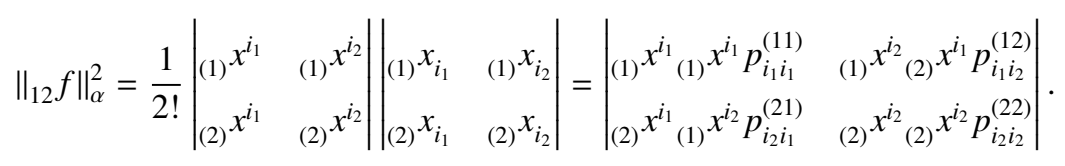

We note that it appears $\frac{1}{2 !}$ in the second side of (47) because we have two determinants. We have therefore two permutations of two indices connected with them. Each determinant of the second side of (47) has evidently two indices. The first determinant has contravariant indices. The second determinant has covariant indices. Hence, we are in need of returning to "normality" by means of $\frac{1}{2 !}$. Conversely, it does not appear $\frac{1}{2 !}$ in the third side of (47) because we have only one determinant. We observe that the first element of the first row of the third side of (47) is obtained by multiplying ${ }_{(1)} x^{i_{1}}$ by ${ }_{(1)} x_{i_{1}}$. We obtain the second element of the first row of the third side of (47) by multiplying ${ }_{(1)} x^{i_{2}}$ by ${ }_{(2)} x_{i_{2}}$. We obtain the first element of the second row of the third side of (47) by multiplying ${ }_{(2)} x^{i_{1}}$ by ${ }_{(1)} x_{i_{1}}$. We obtain the second element of the second row of the third side of (47) by multiplying ${ }_{(2)} x^{i_{2}}$ by ${ }_{(2)} x_{i_{2}}$. Having said that, it is possible to write

$$
\left\|_{12} f\right\|_{\alpha}^{2}=\left|\begin{array}{cc}
\left\|_{(1)} \mathbf{x}\right\|_{\alpha}^{2} & { }_{(1)} \mathbf{x} \odot_{(2)} \mathbf{x} \\
\mathbf{( 2 )}_{(1)} \mathbf{x} & \left\|_{(2)} \mathbf{x}\right\|_{\alpha}^{2}
\end{array}\right|
$$

as well as

$$
\left\|_{12} f\right\|_{\alpha}^{2}=\left|\begin{array}{ll}
\mathbf{P}\left({ }_{1} X_{1} X\right) & \mathbf{P}\left({ }_{1} X_{2} X\right) \\
\mathbf{P}\left({ }_{2} X_{1} X\right) & \mathbf{P}\left({ }_{2} X_{2} X\right)
\end{array}\right| .
$$

We have consequently established that the expected return on a portfolio $X_{12}$ of two univariate risky assets coincides with the $\alpha$-norm of ${ }_{12} f$, so we write

$$
\left\|_{12} f\right\|_{\alpha}^{2}=\mathbf{P}\left(X_{12}\right)=\bar{X}_{12}=\mathbf{P}\left({ }_{1} X_{1} X\right) \cdot \mathbf{P}\left({ }_{2} X_{2} X\right)-\mathbf{P}\left({ }_{1} X_{2} X\right) \cdot \mathbf{P}\left({ }_{2} X_{1} X\right) .
$$

Given $X_{12}^{A}$ and $X_{12}^{B}$, where $X_{12}^{A}$ is a portfolio of two univariate risky assets and $X_{12}^{B}$ is another portfolio of two univariate risky assets, if it turns out to be

$$
\mathbf{P}\left(X_{12}^{A}\right)>\mathbf{P}\left(X_{12}^{B}\right)
$$

then $X_{12}^{A}$ is preferred to $X_{12}^{B}$ (Markowitz, 1952). We also say that $X_{12}^{A}$ has a greater subjective utility than $X_{12}^{B}$ (Nau, 2006). We observe that an investor coherently behaves when he maximizes the expected return on a portfolio of two univariate risky assets (Johnson, \& Payne, 1985). Conversely, if it turns out to be

$$
\mathbf{P}\left(X_{12}^{B}\right)>\mathbf{P}\left(X_{12}^{A}\right)
$$

then $X_{12}^{B}$ is preferred to $X_{12}^{A}$ (MacCrimmon, 1968). We therefore say that $X_{12}^{B}$ has a greater subjective utility than $X_{12}^{A}$. We lastly write

$$
{ }_{(2)} S^{(2) \wedge} \subset E_{(2)}^{m \wedge},
$$

where the linear space of the antisymmetric tensors of order 2 is denoted by $E_{(2)}^{m \wedge}$. 


\section{A Portfolio of Two Univariate Risky Assets and Its Variance}

Given $X=\left\{x^{1}, x^{2}, \ldots, x^{m}\right\}$, its expected return is expressed by means of the vector $\overline{\mathbf{x}}=\left(\bar{x}^{i}\right)$ whose contravariant components are all equal. It allows of defining a univariate risky asset representing deviations denoted by ${ }_{X} d$. This univariate risky asset is evidently defined over $X$ by means of $\mathbf{P}(X)$. We write

$$
{ }_{\mathbf{x}} \mathbf{d}=\mathbf{x}-\overline{\mathbf{x}},
$$

where ${ }_{\mathbf{x}} \mathbf{d}$ is an $m$-dimensional vector of $E^{m}$. Its contravariant components are given by

$$
d^{i}=x^{i}-\bar{x}^{i} .
$$

We deal with a linear transformation of $X$. It is a change of origin. We observe that the expected return on ${ }_{X} d$ is necessarily given by

$$
\mathbf{P}\left({ }_{X} d\right)=\left(x^{i}-\bar{x}^{i}\right) p_{i}=0 .
$$

The $\alpha$-norm of the vector representing ${ }_{X} d$ is given by

$$
\|\mathbf{d}\|_{\alpha}^{2}=\left({ }_{\mathbf{x}} d^{i}\right)^{2} p_{i}=\sigma_{X}^{2} .
$$

It geometrically represents an estimate of the variance of $X$. It represents an estimate of the riskiness of this univariate risky asset. Having said that, we consider two univariate risky assets representing deviations which are respectively ${ }_{1} d$ and ${ }_{2} X$. They are defined over ${ }_{1} X$ and ${ }_{2} X$, where we have $X_{12}=\left\{{ }_{1} X,{ }_{2} X\right\}$. They are represented by the vectors ${ }_{(1)} \mathbf{d}$ and (2) d whose contravariant components are given by

$$
{ }_{(1)} d^{i}={ }_{(1)} x^{i}-{ }_{(1)} \bar{x}^{i}
$$

and

$$
{ }_{(2)} d^{i}={ }_{(2)} x^{i}-{ }_{(2)} \bar{x}^{i}
$$

It is therefore possible to introduce an antisymmetric tensor of order 2 denoted by ${ }_{12} d$. It characterizes ${ }_{X_{12}} d$, where ${ }_{X_{12}} d$ is a portfolio of two univariate risky assets representing deviations. It is defined over $X_{12}$. Its contravariant components are then given by

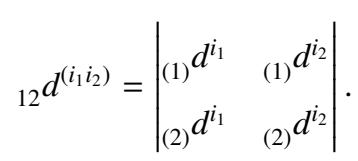

Its covariant components are given by

$$
{ }_{12} d_{\left(i_{1} i_{2}\right)}=\left|\begin{array}{ll}
{ }_{(1)} d_{i_{1}} & { }_{(1)} d_{i_{2}} \\
{ }_{(2)} d_{i_{1}} & \text { (2) } d_{i_{2}}
\end{array}\right|=\left|\begin{array}{ll}
{ }_{(1)} d^{i_{2}} p_{i_{2} i_{1}} & { }_{(1)} d^{i_{1}} p_{i_{1} i_{2}} \\
{ }_{(2)} d^{i_{2}} p_{i_{2} i_{1}} & { }_{(2)} d^{i_{1}} p_{i_{1} i_{2}}
\end{array}\right| .
$$

The $\alpha$-norm of the tensor ${ }_{12} d$ is then expressed by

$$
\left\|_{12} d\right\|_{\alpha}^{2}=\left|\begin{array}{cc}
\left\|_{(1)} \mathbf{d}\right\|_{\alpha}^{2} & { }_{(1)} \mathbf{d} \odot_{(2)} \mathbf{d} \\
\mathbf{( 2 )} \mathbf{d} \odot_{(1)}^{\mathbf{d}} & \left\|_{(2)} \mathbf{d}\right\|_{\alpha}^{2}
\end{array}\right|,
$$

where it turns out to be ${ }_{(1)} \mathbf{d} \odot{ }_{(2)} \mathbf{d}=\operatorname{Cov}\left({ }_{1} X,{ }_{2} X\right)$. We have consequently established that an estimate of the variance of a portfolio $X_{12}$ of two univariate risky assets coincides with the $\alpha$-norm of ${ }_{12} d$, so we write

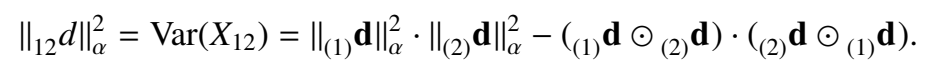

Given $X_{12}^{A}$ and $X_{12}^{B}$, if it turns out to be

$$
\operatorname{Var}\left(X_{12}^{A}\right)>\operatorname{Var}\left(X_{12}^{B}\right)
$$

then $X_{12}^{A}$ is riskier than $X_{12}^{B}$ (Davies, \& Satchell, 2007). We observe that an investor coherently behaves when he minimizes the riskiness of a portfolio of two univariate risky assets (Brooks, \& Zank, 2005). Conversely, if it turns out to be

$$
\operatorname{Var}\left(X_{12}^{B}\right)>\operatorname{Var}\left(X_{12}^{A}\right)
$$

then $X_{12}^{B}$ is riskier than $X_{12}^{A}$ (Wakker, 1994). 


\section{Two Different Quadratic Metrics}

We have established a non-linear metric expressed by (62) characterizing a portfolio of two univariate risky assets denoted by $X_{12}=\left\{{ }_{1} X,{ }_{2} X\right\}$. If $X_{12}$ is a stand-alone entity then it is an antisymmetric tensor of order 2. It is then possible to show that $X_{12}$ coincides with a particular geometric shape. We will show that a non-linear metric is invariant with respect to two different translations concerning ${ }_{(1)} \mathbf{d}$ and ${ }_{(2)} \mathbf{d}$. These two different translations are obtained by considering two different arbitrary $m$-dimensional vectors of $E^{m}$. Also, it is even invariant with respect to two different rotations concerning ${ }_{(1)} \mathbf{d}$ and ${ }_{(2)} \mathbf{d}$. These two different rotations are obtained by considering two different orthogonal $m \times m$ matrices. A non-linear metric is different from a linear metric. A linear metric is expressed by

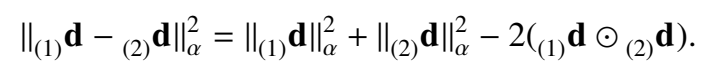

Such a metric does not deal with $X_{12}$ when it is viewed as a stand-alone entity but it deals with two univariate risky assets when they are the marginal univariate components of $X_{12}$ (Abdellaoui, Bleichrodt, \& Paraschiv, 2007). These two univariate risky assets are ${ }_{1} X$ and ${ }_{2} X$. We therefore observe that $X_{12}$ is defined through its marginal univariate components when we speak about a linear metric expressed by (66) (Bernard, \& Ghossoub, 2010). We will show that a linear metric is invariant with respect to two different translations concerning ${ }_{(1)} \mathbf{d}$ and ${ }_{(2)} \mathbf{d}$. These two different translations are obtained by considering two different arbitrary $m$-dimensional vectors of $E^{m}$. It is not invariant with respect to two different rotations concerning ${ }_{(1)} \mathbf{d}$ and ${ }_{(2)} \mathbf{d}$. These two different rotations are obtained by considering two different orthogonal $m \times m$ matrices. We have to note a very important point: a non-linear metric as well as a linear metric are both quadratic because it is not mathematically appropriate to define a non-quadratic metric. We consequently say that a portfolio of more than two univariate risky assets is always characterized by more than one antisymmetric tensor of order 2, where every antisymmetric tensor of order 2 represents a stand-alone bivariate entity. What we have said is then more general than one might think at first.

\section{An Intrinsic Property of a Portfolio of Two Univariate Risky Assets}

Given a univariate risky asset denoted by $X$, we consider the following

Theorem 1 Let $\mathbf{x}^{\prime}=\mathbf{x}-g(\mathbf{x})$ be a vector of $E^{m}$, where $g$ is a linear transformation from $E^{m}$ to $E^{m}$ whose associated matrix is non-singular. Given $\mathbf{z} \in E^{m}$, let $\mathcal{T}_{\mathbf{z}}(\mathbf{x})$ and $\mathcal{T}_{\mathbf{z}}\left(\mathbf{x}^{\prime}\right)$ be two translations with respect to it. If it turns out to be $\mathbf{z}=g(\mathbf{z})$ then $\mathbf{x}^{\prime} \mathbf{d}^{\prime}=\mathbf{x}-g(\mathbf{x}) \in E^{m}$ is invariant under translation.

Proof. We have

$$
\mathcal{T}_{\mathbf{z}}(\mathbf{x}): \mathbf{x} \Rightarrow \mathbf{x}+\mathbf{z}
$$

as well as

$$
\mathcal{T}_{\mathbf{z}}\left(\mathbf{x}^{\prime}\right): \mathbf{x}^{\prime} \Rightarrow \mathbf{x}^{\prime}+\mathbf{z}
$$

They are two translations with respect to $\mathbf{z} \in E^{m}$, where $\mathbf{z}$ is a vector of $E^{m}$ that we choose. Given

$$
\mathbf{x}^{\prime}=\mathbf{x}-g(\mathbf{x})
$$

it is then possible to write

$$
\mathcal{T}_{\mathbf{z}}\left(\mathbf{x}^{\prime}\right)=\mathcal{T}_{\mathbf{z}}[\mathbf{x}-g(\mathbf{x})]=\mathcal{T}_{\mathbf{z}}(\mathbf{x})-\mathcal{T}_{\mathbf{z}}[g(\mathbf{x})] .
$$

Since $g$ is a linear transformation, we are able to write

$$
\mathcal{T}_{\mathbf{z}}\left(\mathbf{x}^{\prime}\right)=\mathcal{T}_{\mathbf{z}}(\mathbf{x})-g\left[\mathcal{T}_{\mathbf{z}}(\mathbf{x})\right]
$$

The translation that appears as a minuend in (71) is given by (67). We have to define the translation that appears as a subtrahend in (71): we write

$$
g\left[\mathcal{T}_{\mathbf{z}}(\mathbf{x})\right]: g(\mathbf{x}) \Rightarrow g(\mathbf{x}+\mathbf{z})
$$

We therefore obtain

$$
\mathcal{T}_{\mathbf{z}}\left(\mathbf{x}^{\prime}\right): \mathbf{x}-g(\mathbf{x}) \Rightarrow \mathbf{x}+\mathbf{z}-g(\mathbf{x}+\mathbf{z})
$$

after considering (67) and (72) in (68). We have to establish which is that condition according to which it is possible to say that $\mathbf{x}^{\prime}=\mathbf{x}-g(\mathbf{x})$ is invariant under translation. It is then expressed by

$$
\mathbf{x}-g(\mathbf{x})=\mathbf{x}+\mathbf{z}-g(\mathbf{x}+\mathbf{z}) .
$$

Since $g$ is a linear transformation we write

$$
\mathbf{x}-g(\mathbf{x})=\mathbf{x}+\mathbf{z}-g(\mathbf{x})-g(\mathbf{z}) .
$$


We therefore say that $\mathbf{x}^{\prime}$ is invariant under translation because it turns out to be

$$
\mathbf{z}=g(\mathbf{z})
$$

The equality expressed by (76) exists when $g$ is a linear transformation viewed as an identity function applied to $E^{m}$. It exists when $\mathbf{z}$ is the zero vector of $E^{m}$. On the other hand, these two cases can be viewed as degenerate cases. It also exists when $\mathbf{z}$ is a constant. It therefore exists when $\mathbf{z}$ represents the expected return on $X$, so its contravariant components are all equal. Hence, from (69) it follows that it is possible to write

$$
{ }_{\mathbf{x}} \mathbf{d}^{\prime}={ }_{\mathbf{x}} \mathbf{d}-g\left({ }_{\mathbf{x}} \mathbf{d}\right)
$$

where it turns out to be ${ }_{\mathbf{x}} \mathbf{d}=\mathbf{x}-\overline{\mathbf{x}}$. We consequently write

$$
\mathbf{x}^{\prime}=(\mathbf{x}-\overline{\mathbf{x}})-g(\mathbf{x}-\overline{\mathbf{x}})=\mathbf{x}-\overline{\mathbf{x}}-g(\mathbf{x})+g(\overline{\mathbf{x}}) .
$$

Since we have $g(\overline{\mathbf{x}})=\overline{\mathbf{x}}$ it turns out to be

$$
\mathbf{x}^{\prime}=\mathbf{x}-g(\mathbf{x}) .
$$

This means that (79) coincides with the vector $\mathbf{x}^{\prime}$ expressed by (69). We have proved that (79) is that vector which is invariant under translation. It represents all deviations of $\mathbf{x}$ from $\overline{\mathbf{x}}$.

We consider the following

Corollary If $\mathbf{y}$ is a vector of $E^{m}$ freely chosen then it turns out to be $\mathcal{T}_{\mathbf{y}}\left({ }_{\mathbf{x}} \mathbf{d}\right)=\mathcal{T}_{\mathbf{y}}\left({ }_{\mathbf{x}} \mathbf{d}^{\prime}\right)={ }_{\mathbf{x}} \mathbf{d}$.

Proof. We write

$$
\mathcal{T}_{\mathbf{y}}\left({ }_{\mathbf{x}} \mathbf{d}\right):{ }_{\mathrm{x}} \mathbf{d} \Rightarrow{ }_{\mathrm{x}} \mathbf{d}+\mathbf{y}=\mathbf{x}-\overline{\mathbf{x}}+\mathbf{y}
$$

with regard to ${ }_{\mathbf{x}} \mathbf{d}$. We write

$$
\mathcal{T}_{\mathbf{y}}\left({ }_{\mathbf{x}} \mathbf{d}^{\prime}\right):{ }_{\mathbf{x}} \mathbf{d}^{\prime} \Rightarrow{ }_{\mathbf{x}} \mathbf{d}^{\prime}+\mathbf{y}=\mathbf{x}-g(\mathbf{x})+\mathbf{y}
$$

with regard to ${ }_{\mathbf{x}} \mathbf{d}^{\prime}$. By making equal (80) and (81) it turns out to be

$$
\mathbf{x}-\overline{\mathbf{x}}+\mathbf{y}=\mathbf{x}-g(\mathbf{x})+\mathbf{y} .
$$

We observe that we write $g(\mathbf{x})=\overline{\mathbf{x}}$, so the vector representing deviations is invariant under translation. We observe that (82) does not depend on $\mathbf{y} \in E^{m}$, where $\mathbf{y}$ is a vector of $E^{m}$ that we freely choose. We lastly write

$$
\mathcal{T}_{\mathbf{y}}\left({ }_{\mathbf{x}} \mathbf{d}\right)=\mathcal{T}_{\mathbf{y}}\left({ }_{\mathbf{x}} \mathbf{d}^{\prime}\right)={ }_{\mathbf{x}} \mathbf{d}
$$

We have then proved how an $m$-dimensional vector of $E^{m}$ denoted by ${ }_{\mathbf{x}} \mathbf{d}$ is invariant under translation.

What we have said can evidently be extended to ${ }_{(1)} \mathbf{d}$ and ${ }_{(2)} \mathbf{d}$. Given two arbitrary vectors $\mathbf{u}$ and $\mathbf{t}$ of $E^{m}$, we say that it is possible to write

$$
\left\|_{(1)} \mathbf{d}-{ }_{(2)} \mathbf{d}\right\|_{\alpha}^{2}=\left\|\mathcal{T}_{\mathbf{u}}\left(_{(1)} \mathbf{d}\right)-\mathcal{T}_{\mathbf{t}}\left(_{(2)} \mathbf{d}\right)\right\|_{\alpha}^{2}
$$

according to (83). This means that a linear metric is invariant with respect to two different translations concerning ${ }_{(1)} \mathbf{d}$ and ${ }_{(2)} \mathbf{d}$. We note that the contravariant components of $\mathbf{u}$ with respect to an orthonormal basis of $E^{m}$ are all equal. The same thing goes with respect to the contravariant components of $\mathbf{t}$. If (83) holds then we are able to say that a non-linear metric expressed by (62) is invariant with respect to two different translations concerning (1) $\mathbf{d}_{\text {and }} \mathbf{d}$. We are able to consider different translations concerning the possible monetary values of each risky asset contained in a portfolio. It is then possible to deal with different portfolios of risky assets whose riskiness is the same although their expected return is not the same.

\section{Another Intrinsic Property of a Portfolio of Two Univariate Risky Assets}

Given ${ }_{\mathbf{x}} \mathbf{d} \in E^{m}$, we wonder what it happens if ${ }_{\mathbf{x}} \mathbf{d}$ is subjected to a rotation by using $A$, where $A=\left(a_{j}^{i^{\prime}}\right)$ is an $m \times m$ matrix. It is an orthogonal matrix whose upper index represents its rows. Its columns are represented by the $j$ index. We write

$$
\mathcal{R}_{A}\left({ }_{\mathbf{x}} \mathbf{d}\right):{ }_{\mathbf{x}} \mathbf{d} \Rightarrow A\left({ }_{\mathbf{x}} \mathbf{d}\right)={ }_{\mathbf{x}}^{R} \mathbf{d}
$$

where ${ }_{\mathbf{x}}^{R} \mathbf{d}$ is an $m$-dimensional rotated vector belonging to $E^{m}$. It is possible to show that its contravariant and covariant components are different from the ones of ${ }_{\mathbf{x}} \mathbf{d}$. We observe that (56) does not invariably hold when it regards ${ }^{R} X$, where ${ }^{R} X$ denotes that $X$ has been rotated. We note that it suffices to consider all its deviations. We are therefore able to say that 
${ }^{R} X$ and $X$ are two different univariate risky assets. It suffices to consider their deviations only. On the other hand, it is possible to show that their variance is the same, so we write

$$
\left\|\mathcal{R}_{A}\left({ }_{\mathbf{x}} \mathbf{d}\right)\right\|_{\alpha}^{2}=\|\mathbf{x} \mathbf{d}\|_{\alpha}^{2} .
$$

Having said that, we refer to a portfolio of two univariate risky assets when it is viewed as an antisymmetric tensor of order 2. We consider all deviations of these two univariate risky assets. We show the following

Theorem 2 If ${ }_{(1)} \mathbf{d} \in E^{m}$ and ${ }_{(2)} \mathbf{d} \in E^{m}$ are subjected to two different rotations respectively expressed by $A=\left(a_{i_{2}}^{i_{1}{ }^{\prime}}\right)$ and $B=\left(b_{i_{2}}^{i_{1}{ }^{\prime}}\right)$ then it turns out to be $\left\|_{12}^{R} d\right\|_{\alpha}^{2}=\left\|_{12} d\right\|_{\alpha}^{2}$.

Proof. We consider

$$
{ }_{(1)}^{R} d^{i_{1}{ }^{\prime}}={ }_{(1)} d^{i_{2}} a_{i_{2}}^{i_{1}{ }^{\prime}},
$$

where we deal with $m$ linear and homogeneous relationships that allow us of passing from the contravariant components of a type of vector to the ones of another type of vector. This is because $A$ is an $m \times m$ matrix. It is an orthogonal matrix. On the other hand, we consider

$$
{ }_{(2)}^{R} d^{i_{1}{ }^{\prime}}={ }_{(2)} d^{i_{2}} b_{i_{2}}^{i_{1}{ }^{\prime}},
$$

where we deal with $m$ linear and homogeneous relationships that allow us of passing from the contravariant components of a type of vector to the ones of another type of vector. This is because $B$ is an $m \times m$ matrix. It is an orthogonal matrix. We also consider

$$
{ }_{(1)}^{R} d_{i_{1}{ }^{\prime}}={ }_{(1)} d_{i_{2}} a_{i_{1}{ }^{\prime}}^{i_{2}},
$$

where we deal with $m$ linear and homogeneous relationships that allow us of passing from the covariant components of a type of vector to the ones of another type of vector. In general, we take the product between each value of a vector representing deviations and its corresponding probability into account when we consider its covariant components. We deal with the transpose of $A$ in (89). We have

$$
{ }_{(2)}^{R} d_{i_{1}{ }^{\prime}}={ }_{(2)} d_{i_{2}} b_{i_{1^{\prime}}}^{i_{2}}
$$

as well, where we deal with $m$ linear and homogeneous relationships that allow us of passing from the covariant components of a type of vector to the ones of another type of vector. In general, we take the product between each value of a vector representing deviations and its corresponding probability into account when we consider its covariant components. We deal with the transpose of $B$ in (90). Having said that, we already know that it is possible to write

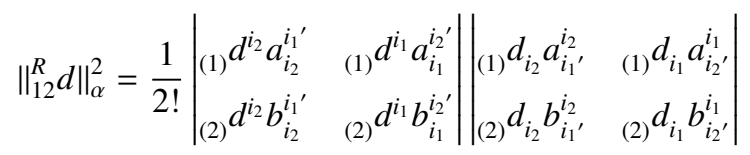

as well as

$$
\left\|_{12}^{R} d\right\|_{\alpha}^{2}=\left|\begin{array}{cc}
\left\|_{(1)} \mathbf{d}\right\|_{\alpha}^{2} & \left(_{(1)} \mathbf{d} \odot_{(2)} \mathbf{d}\right) a_{i_{2}}^{i_{1}{ }^{\prime}} b_{i_{1}{ }^{\prime}}^{i_{2}} \\
\left.{ }_{(2)} \mathbf{d} \odot_{(1)} \mathbf{d}\right) b_{i_{2}}^{i_{1_{1}}} a_{i_{1^{\prime}}}^{i_{2}} & \left\|_{(2)} \mathbf{d}\right\|_{\alpha}^{2}
\end{array}\right|,
$$

so it turns out to be

$$
\begin{aligned}
\left\|_{12}^{R} d\right\|_{\alpha}^{2} & =\left\|_{(1)} \mathbf{d}\right\|_{\alpha}^{2} \cdot\left\|_{(2)} \mathbf{d}\right\|_{\alpha}^{2}-\left(_{(1)} \mathbf{d} \odot_{(2)} \mathbf{d}\right)^{2} a_{i_{2}}^{i_{1}{ }^{\prime}} b_{i_{1}}^{i_{2}} b_{i_{2}}^{i_{1}{ }^{\prime}} a_{i_{1}}^{i_{2}{ }^{\prime}} \\
& =\left\|_{(1)} \mathbf{d}\right\|_{\alpha}^{2} \cdot\left\|_{(2)} \mathbf{d}\right\|_{\alpha}^{2}-\left(_{(1)} \mathbf{d} \odot_{(2)} \mathbf{d}\right)^{2}\left(a_{i_{2}}^{i_{1}} a_{i_{1_{1}}}^{i_{2}}\right)\left(b_{i_{1}}^{i_{2}}, b_{i_{2}}^{i_{1}{ }^{\prime}}\right) \\
& =\left\|_{(1)} \mathbf{d}\right\|_{\alpha}^{2} \cdot\left\|_{(2)} \mathbf{d}\right\|_{\alpha}^{2}-\left(_{(1)} \mathbf{d} \odot_{(2)} \mathbf{d}\right)^{2}
\end{aligned}
$$

because $A$ and $B$ are both orthogonal. We lastly write

$$
\left\|_{12}^{R} d\right\|_{\alpha}^{2}=\left\|_{12} d\right\|_{\alpha}^{2} .
$$

We have therefore proved that a non-linear metric is invariant with respect to two different rotations concerning ${ }_{(1)} \mathbf{d}$ and (2) d.

On the other hand, it is possible to show that a linear metric is not invariant with respect to two different rotations concerning ${ }_{(1)} \mathbf{d}$ and ${ }_{(2)} \mathbf{d}$. We note that it is possible that ${ }_{\mathrm{x}}^{R} \mathbf{d}$ contains fewer possible and distinct values than ${ }_{\mathrm{x}} \mathbf{d}$. We therefore observe that the corresponding probabilities of the remaining and distinct values of ${ }_{\mathbf{x}}^{R} \mathbf{d}$ increase. On the other hand, ${ }_{\mathbf{x}}^{R} \mathbf{d}$ and ${ }_{\mathbf{x}} \mathbf{d}$ always represent two univariate risky assets whose riskiness is the same. The same thing evidently goes when we consider ${ }_{(1)}^{R} \mathbf{d}$ and ${ }_{(1)} \mathbf{d}$ as well as ${ }_{(2)}^{R} \mathbf{d}$ and ${ }_{(2)} \mathbf{d}$. We are consequently able to consider different portfolios of univariate risky assets whose riskiness is the same. 


\section{Conclusions}

We have shown a canonical expression of a univariate risky asset. We have found out a canonical expression of the product of two univariate risky assets when they are jointly considered. We have found out a canonical expression of a portfolio of two univariate risky assets when it is viewed as a stand-alone entity. We have proved that a univariate risky asset is an isometry. We have defined different distributions of probability on $\mathbb{R}$ inside of metric spaces having different dimensions. We have used the geometric property of collinearity in order to obtain this thing. We have computed the expected return on a portfolio of two univariate risky assets when it is viewed as an antisymmetric tensor of order 2 . We have also computed its variance. We have considered two intrinsic properties of a portfolio of it. What we have said can be extended to a portfolio of more than two univariate risky assets.

\section{References}

Abdellaoui, M., Bleichrodt, H., \& Paraschiv, C. (2007). Loss aversion under prospect theory: a parameter-free measurement. Management Science, 53(10), 1659-1674. https://doi.org/10.1287/mnsc.1070.0711

Anscombe, F. J., \& Aumann, R. J. (1963). A definition of subjective probability. The Annals of Mathematical Statistics, 34(1), 199-205. https://doi.org/10.1214/aoms/1177704255

Berkelaar, A. B., Kouwenberg, R., \& Post, T. (2004). Optimal portfolio choice under loss aversion. Review of Economics and Statistics, 86(4), 973-987. https://doi.org/10.1162/0034653043125167

Bernard, C., \& Ghossoub, M. (2010). Static portfolio choice under cumulative prospect theory. Mathematics and Financial Economics, 2, 277-306.

Berti, P., Regazzini, E., \& Rigo, P. (2001). Strong previsions of random elements. Statistical Methods and Applications (Journal of the Italian Statistical Society), 10, 11-28. https://doi.org/10.1007/BF02511636

Berti, P., Pratelli, L., \& Rigo, P. (2015). Two versions of the fundamental theorem of asset pricing. Electronic Journal of Probability, 20, 1-21. https://doi.org/10.1214/EJP.v20-3321

Brooks, P., \& Zank, H. (2005). Loss averse behavior. Journal of Risk and Uncertainty, 31, $301-325$. https://doi.org/10.1007/s11166-005-5105-7

Coletti, G., Petturiti, D., \& Vantaggi, B. (2016a). When upper conditional probabilities are conditional possibility measures. Fuzzy Sets and Systems, 304, 45-64. https://doi.org/10.1016/j.ins.2015.12.020

Coletti, G., Petturiti, D., \& Vantaggi, B. (2016b). Conditional belief functions as lower envelopes of conditional probabilities in a finite setting. Information Sciences, 339, 64-84.

Coletti, G., Scozzafava, R., \& Vantaggi, B. (2015). Possibilistic and probabilistic logic under coherence: default reasoning and System P. Mathematica Slovaca, 65(4), 863-890. https://doi.org/10.1515/ms-2015-0060

Coletti, G., Petturiti, D., \& Vantaggi, B. (2014). Bayesian inference: the role of coherence to deal with a prior belief function. Statistical Methods $\mathcal{E}$ Applications, 23(4), 519-545. https://doi.org/10.1007/s10260-014-0279-2

de Finetti, B. (1982a). The proper approach to probability. In G. Koch, \& F. Spizzichino (Eds.), Exchangeability in Probability and Statistics (pp. 1-6), North-Holland Publishing Company, Amsterdam.

de Finetti, B. (1972a). Probability, Statistics and Induction: their relationship according to the various points of view. In B. de Finetti (Ed.), Probability, Induction and Statistics (The art of guessing) (pp. 147-227). J. Wiley, London-New York-Sydney-Toronto.

de Finetti, B. (1989). Probabilism: A Critical Essay on the Theory of Probability and on the Value of Science. Erkenntnis, 31(2-3), 169-223. https://doi.org/10.1007/BF01236563

de Finetti, B. (1964). Foresight: its logical laws, its subjective sources. In H. E. Kyburg jr., \& H. E. Smokler (Eds.), Studies in subjective probability (pp. 93-158). J. Wiley, New York.

de Finetti, B. (1972b). How to choose the initial probabilities. In B. de Finetti (Ed.), Probability, Induction and Statistics (The art of guessing) (pp. 143-146). J. Wiley, London-New York-Sydney-Toronto.

de Finetti, B. (1980). Probability: beware of falsifications!. In H. E. Kyburg jr., \& H. E. Smokler (Eds.), Studies in subjective probability (pp. 195-224). R. E. Krieger Publishing Company, Huntington, New York. 
de Finetti, B. (1981). The role of "Dutch Books" and of "proper scoring rules". The British Journal of Psychology of Sciences, 32, 55-56. https://doi.org/10.1093/bjps/32.1.55

de Finetti, B. (1982b). Probability: the different views and terminologies in a critical analysis. In L. J. Cohen, J. Łoś, H. Pfeiffer, \& K.-P. Podewski (Eds.), Logic, Methodology and Philosophy of Science VI (pp. 391-394). North-Holland Publishing Company, Amsterdam. https://doi.org/10.1016/S0049-237X(09)70208-3

Davies, G. B., \& Satchell, S. E. (2007). The behavioural components of risk aversion. Journal of Mathematical Psychology, 5l(1), 1-13. https://doi.org/10.1016/j.jmp.2006.10.003

deGroot, M. H. (1962). Uncertainty, information and sequential experiments. The Annals of Mathematical Statistics, 33, 404-419. https://doi.org/10.1214/aoms/1177704567

Gilio, A., \& Sanfilippo, G. (2014). Conditional random quantities and compounds of conditionals. Studia logica, 102(4), 709-729. https://doi.org/10.1007/s11225-013-9511-6

Good, I. J. (1962). Subjective probability as the measure of a non-measureable set. In E. Nagel, P. Suppes, \& A. Tarski (Eds.), Logic, Methodology and Philosophy of Science (pp. 319-329). Stanford University Press, Stanford.

He, X. D., \& Zhou, X. Y. (2011). Portfolio choice under cumulative prospect theory: an analytical treatment. Management Science, 57(2), 315-331. https://doi.org/10.2139/ssrn.1479580

Johnson, E. J., \& Payne, J. W. (1985). Effort and accuracy in choice. Management Science, 31(4), 395-414. https://doi.org/10.1287/mnsc.31.4.395

Koopman, B. O. (1940). The axioms and algebra of intuitive probability. Annals of Mathematics, 41, 269-292. https://doi.org/10.2307/1969003

Markowitz, H. (1952). The utility of wealth. Journal of Political Economy, 60(2), 151-158. https://doi.org/10.1086/257177

MacCrimmon, K. R. (1968). Descriptive and normative implications of the decision-theory postulates. In K. Borch, \& J. Mossin (Eds.), Risk and Uncertainty (pp. 3-32). Palgrave Macmillan, London. https://doi.org/10.1007/978-1-34915248-3_1

Nau, R. F. (2006). Uncertainty aversion with second-order utilities and probabilities. Management Science, 52(1), 136145. https://doi.org/10.1287/mnsc.1050.0469

Nunke, R. J., \& Savage, L. J. (1952). On the set of values of a nonatomic, finitely additive, finite measure. Proceedings of the American Mathematical Society, 3(2), 217-218. https://doi.org/10.1090/S0002-9939-1952-0049272-6

Pfanzagl, J. (1967). Subjective probability derived from the Morgenstern-von Neumann utility theory. In M. Shubik (Ed.), Essays in mathematical economics in honor of Oskar Morgenstern (pp. 237-251). Princeton University Press, Princeton. https://doi.org/10.1515/9781400877386-020

Piccinato, L. (1986). De Finetti's logic of uncertainty and its impact on statistical thinking and practice. In P. K. Goel, \& A. Zellner (Eds.), Bayesian Inference and Decision Techniques (pp. 13-30). North-Holland, Amsterdam.

Pompilj, G. (1957). On intrinsic independence. Bulletin of the International Statistical Institute, 35(2), 91-97.

Slovic, P., Fischhoff, B., \& Lichtenstein, S. (1977). Behavioral decision theory. Annual Review of Psychology, 28, 1-39. https://doi.org/10.1146/annurev.ps.28.020177.000245

Tversky, A., \& Kahneman, D. (1992). Advances in prospect theory: cumulative representation of uncertainty. Journal of Risk and Uncertainty, 5(4), 297-323. https://doi.org/10.1007/BF00122574

von Neumann, J. (1936). Examples of continuous geometries. Proceedings of the National Academy of Sciences of the United States of America, 22(2), 101-108. https://doi.org/10.1073/pnas.22.2.101

Wakker, P. (1994). Separating marginal utility and probabilistic risk aversion. Theory and Decision, 36(1), 1-44. https://doi.org/10.1007/BF01075296

Zank, H. (2010). On probabilities and loss aversion. Theory and Decision, 68, 243-261. https://doi.org/10.1007/s11238008-9117-z 


\section{Copyrights}

Copyright for this article is retained by the author(s), with first publication rights granted to the journal.

This is an open-access article distributed under the terms and conditions of the Creative Commons Attribution license (http://creativecommons.org/licenses/by/4.0/). 